\title{
NUMERICAL SIMULATIONS OF EARTHQUAKE SOURCE PROCESS BY A THREE-DIMENSIONAL CRACK MODEL. PART II. SEISMIC WAVES AND SPECTRUM
}

\author{
Takashi MryatakE* \\ Disaster Prevention Research Institute, Kyoto University, Uji, Japan \\ (Received August 18, 1980; Revised December 25, 1980)
}

\begin{abstract}
We study the seismic waves and spectrum generated by a dynamical rupture process by a three-dimensional crack model obtained in Part I (MTYATAKE, 1980). The fracture condition used here is a finite stress fracture criterion, while the strength is assumed to be distributed irregularly. It is found that complex seismic waves are generated by the irregular rupture process rather than by the complex source-time functions. Near-field and far-field seismic wave radiated from the fault with the above distribution are also calculated. Seismic waves from the fault with a line barrier and a block barrier have almost similar wave shapes. Random barrier distribution radiates high frequency seismic waves, and the spectral amplitudes tend to decay inversely in proportion to the frequency in the range beyond the corner frequency.
\end{abstract}

\section{Introduction}

Since a statistical model have been proposed by HASKELL (1966), a scaling relation of earthquakes has been studied in detail (AKI, 1967, 1972; KANAMORI and Anderson, 1975; Geller, 1976; Ben-Menahem, 1977). AKI (1967) first proposed a simple scaling law based on the assumption that large and small earthquakes have similarities, i.e., the same geometry, a constant stress drop, constant rupture velocity and constant slip velocity. In this model the spectral shape of seismic waves from all earthquakes has a similar form, i.e., the spectrum intensity decays inversely in proportion to the square of frequency in the range beyond the corner frequency. AKI (1972) later revised this model to some extent. KANAMORI and ANDERSON (1975) obtained a scaling relation using a dislocation function, whereas Aki's approach is based on the autocorrelation of particle velocity. By using coda waves, CHOUET et al. (1978) clarified that the departure from scaling law of AKI (1967) cannot be explained by Akr's revised model (1972). This requires more revision of the scaling laws.

The generation mechanism of high frequency seismic waves and its contri-

* Present address: Earthquake Prediction Data Center, Earthquake Research Institute, University of Tokyo, Tokyo, Japan 
butions to the scaling laws has not been discussed so far in relation to fault properties. In this paper, we calculate near-field, far-field seismic waves and spectrum from the model of Part I, for the purpose of investigating the relationship between the irregularity of strength and seismic waves, and also the generation process of multiple shocks.

\section{Seismic Waves}

We shall here investigate the radiation of seismic waves from a fault with an irregular distribution of frictional strength. A two-dimensional crack approach was made by DAS and AKr (1977a, b) who studied far-field seismic waves emitted from a barrier model. For a three-dimensional case, Mikumo and Miyatake (1978) have calculated near-field seismic waves from a frictional faulting model. But this model is not completely three-dimensional, but quasi-three-dimensional only. The model still involves some problems for absolute amplitudes and frequencies for the radiated seismic waves to be studied intensively. In Part I, we investigate the dynamical rupture process by a three-dimensional crack model combined with finite stress fracture criterion.

In the present paper, we deal with seismic waves in a very near-field above the fault, and in near-fields in an ordinary sense, as well as with far-field seismic waves. And we investigate possible relations between irregularities of the fault strength and the waveform and spectrum of seismic waves. Irregularities used here are the same as those in Part I, i.e., a line barrier which divides the fault into two parts, a square-shaped block barrier in the center of the fault, and a random barrier distribution.

\subsection{Very near-field seismic waves immediately above the fault}

Seismic waves in the near-field just on the fault (at $z=0$ plane) is calculated by the finite difference method. Because of the strike-slip fault assumed here, the displacement vector just above the fault has only the $z$-component perpendicular to the fault plane, and hence the ground motion here is generated mainly by the $\mathrm{S}$ waves. In Part I, we calculated rupture propagation for many different types of strength distribution. But only four representative examples, i.e., a uniform strength, cases with a line barrier (Case LB-1 in Part I), with a block barrier, and a random distribution of the strength (Case D-2 in Part I) are here selected. The waves calculated for these cases have been smoothed by a high-cut filter with a cut-off period of $0.5 \mathrm{sec}$ taking the accuracy of the finite difference method described in Part I into account.

\section{a) Uniform strength (Fig. 1)}

In this case, we notice that a phase of large amplitude appears at 2-5 sec after the first arrival. These times nearly correspond to those at which the $\mathrm{S}$ waves radiating from a fault segment nearest to the observing point arrives. It is im- 
portant to note that even in coherently propagating rupture over a fault with a uniform strength, the passage of the rupture nearest to the station produces a large-amplitude phase as if it were a single earthquake. Strictly speaking, the large phase is not generated by the fracture of the nearest fault segment itself, but by the passage of the propagating rupture, because the observing point in this case is located near a nodal line of the $\mathrm{S}$ waves.

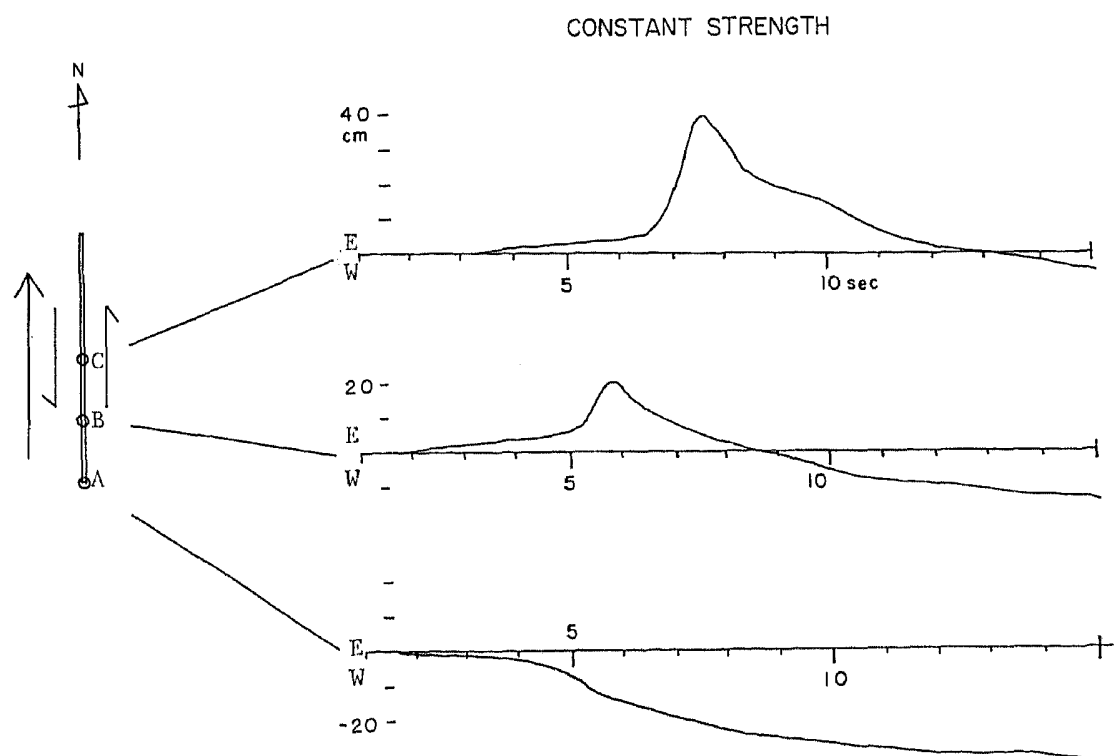

Fig. 1. Near-field displacements at points A, B and C above the fault plane in the case of a uniform strength.

\section{b) Line barrier (Fig. 2, Case LB-1)}

The wave shapes at points $A, B$ and $C$ appear similar to those in Case a) up to about $9 \mathrm{sec}$, but it is seen that at point B after $10 \mathrm{sec}$ and at point $\mathrm{A}$ after $11 \mathrm{sec}$, almost identical phases appeared. We surmise that these phases are generated by the passage of the rupture around the barrier region. Another remarkable phase that appears at about $15 \mathrm{sec}$ may be generated by fracturing of the barriers themselves. Thus we can see three phases, i.e., the first arrival from the starting point of rupture, the second phase generated by the passage of the rupture near the observing point, and the third by the fracture of the barrier region.

c) Block barrier (Fig. 3)

In this case we can see similar features of the wave arrivals to the case of a line barrier up to $9 \mathrm{sec}$, but after this time the sharp phases generated by the barrier cannot be clearly noticed. We think that this is caused by the fact that the distance from the barrier to the observing point becomes considerably large and so the effects due to the barrier is smaller than those in Case b). 


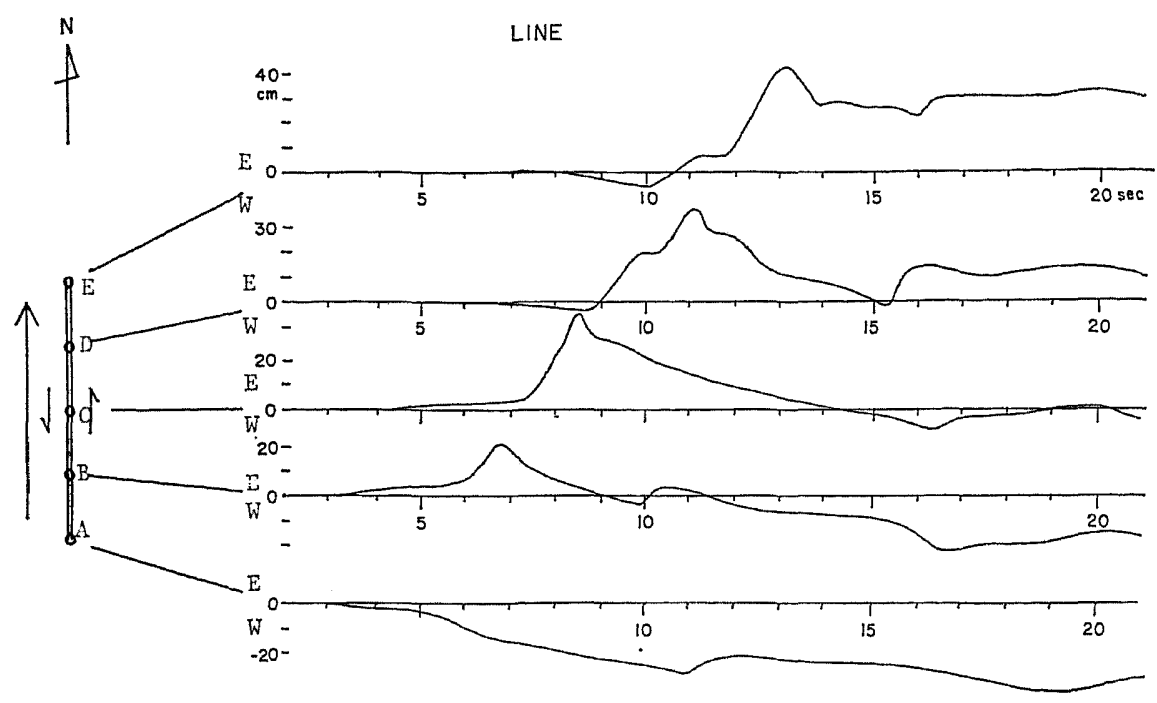

Fig. 2. Same as Fig. 1 for line barrier case (LB-1).

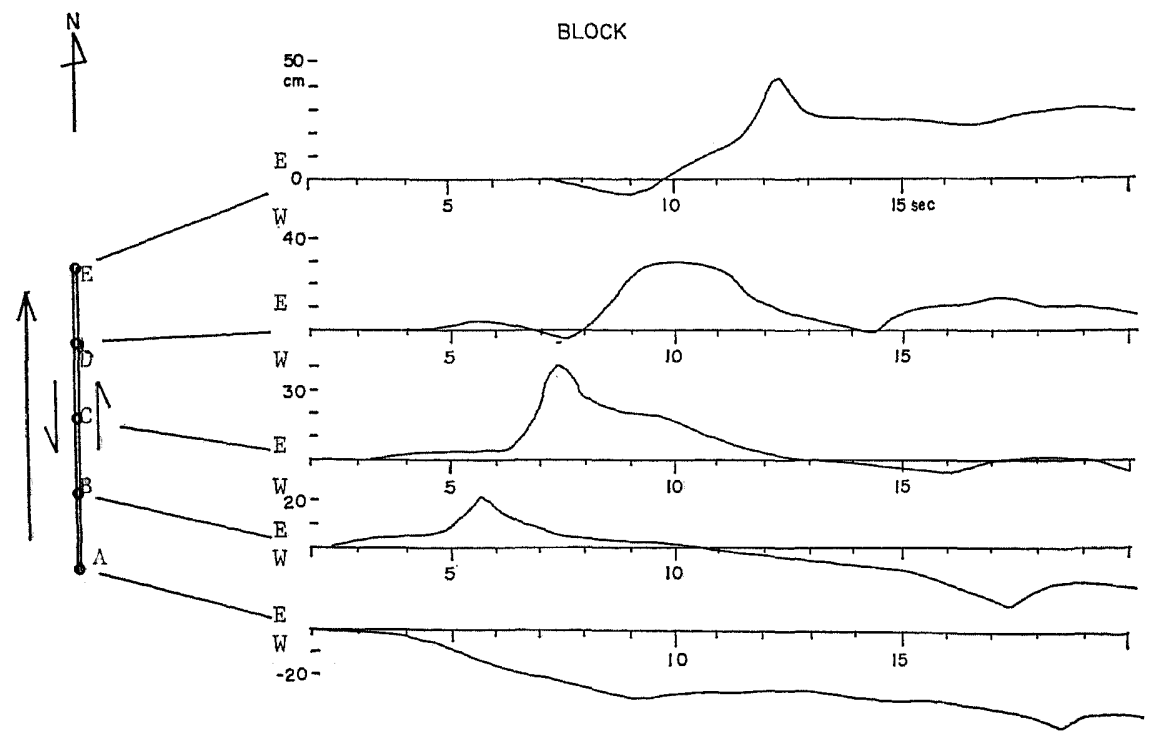

Fig. 3. Same as Fig. 1 for block barrier case.

\section{d) Random strengths (Fig. 4, Case D-2)}

A fault with a random strength distribution generates complex seismic waves, which contain high-frequency components superposing on the low frequency waves as found in the case of a uniform strength. It is noticed that the waveform at point $B$ is remarkably different from that in Case a). This may be understood from the 


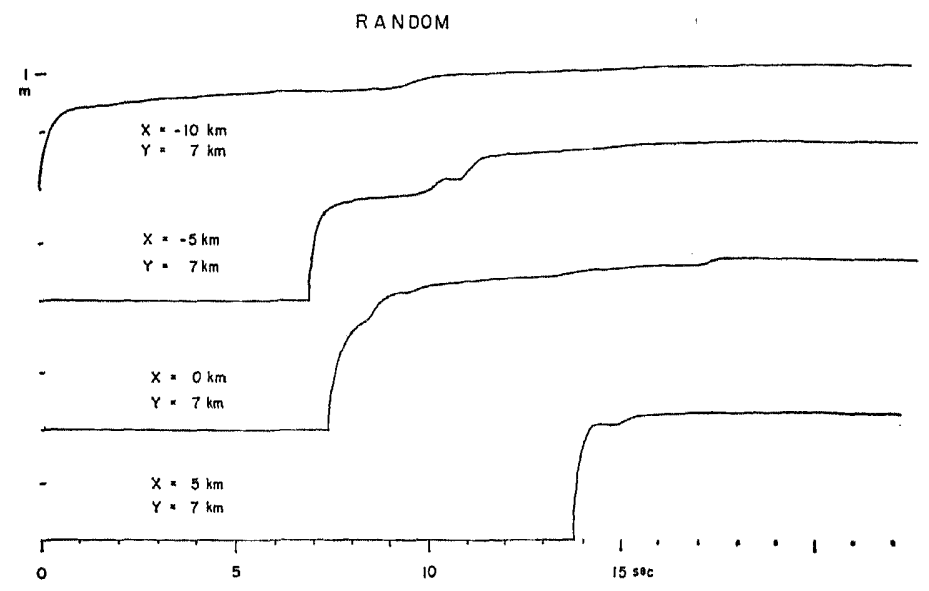

Fig. 4. Same as Fig. 1 for random barrier strength case (D-2).

rupture pattern shown in Fig. 13 (Part I). When we look at the rupture pattern near the fault segment close to point $B$, the rupture appears to propagate bilaterally since a barrier region in the upper left is broken at some time later. The rupture propagation in this case can not generate a sufficient energy of the $\mathrm{S}$ waves at point B.

We summarize the following results for a very near-field seismic waves:

1) Seismic waves immediately above the fault are largely affected by the fracture of a fault segment nearest to the observing point.

2) When the rupture passes by this point, a large sharp phase appears. The rupture velocity may be estimated from the arrival time of the phase.

3) Seismic waveforms become complicated due to the existence of a barrier. An estimate of the barrier's shape and its location from these phases is a problem left unsolved. If we can get data at many points very close to the fault, however, it might be possible to find the existence of a barrier and estimate its location from irregularities of large phases described in the above.

\subsection{Near field seismic waves}

We shall next investigate seismic waves in the near-field which spreads over an area comparable to the fault dimension. We calculate near-field waves from the representations of MARUYAMA (1963) for dynamic dislocations in an infinite space. Here we assign the respective source time functions which have been obtained by the finite difference method in Part I, to each fault element. Seismic waves are obtained at a distance of $50 \mathrm{~km}$ for azimuthal angles of $0,30,60,90$, 120,150 and $180^{\circ}$, which are measured clockwise, respectively. The strength distributions on the fault plane are again of the four types, i.e., uniform strength, cases with a line barrier, with a block barrier, and random distribution of the 
strength. The results obtained are described below. In the following figures, the absolute displacement amplitudes are not indicated, since we are mainly concerned with the change in the waveform in different directions. The calculated maximum amplitudes are of the order of $4 \mathrm{~cm}$.

a) Uniform strength (Figs. 5(a) and (b))

First, we calculate near-field seismic waves emitted from a uniform strength fault, for later comparison with those from other types of strength distributions. Figures 5(a) and (b) show the $x$ - and $z$-displacement components at several points in the near-field.

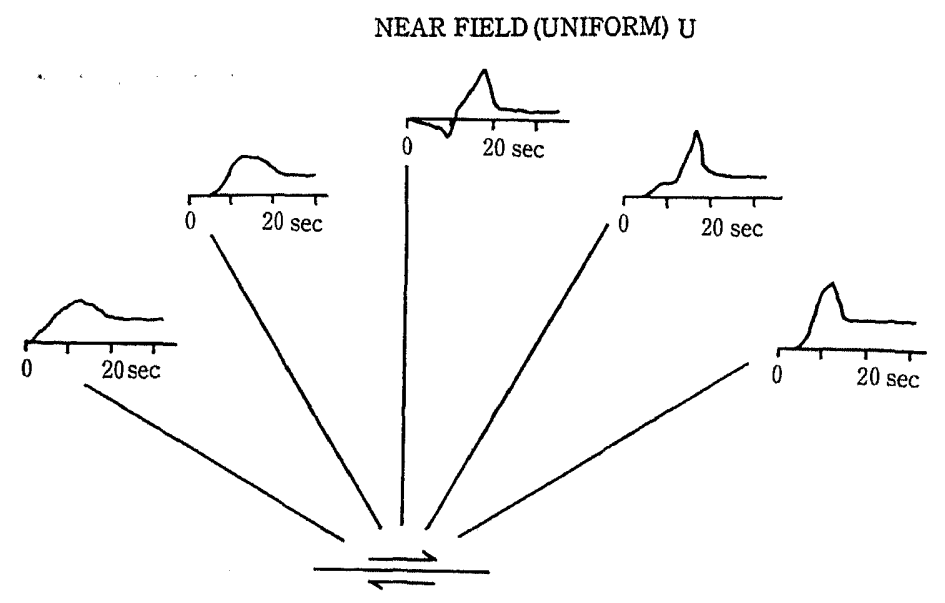

(a)

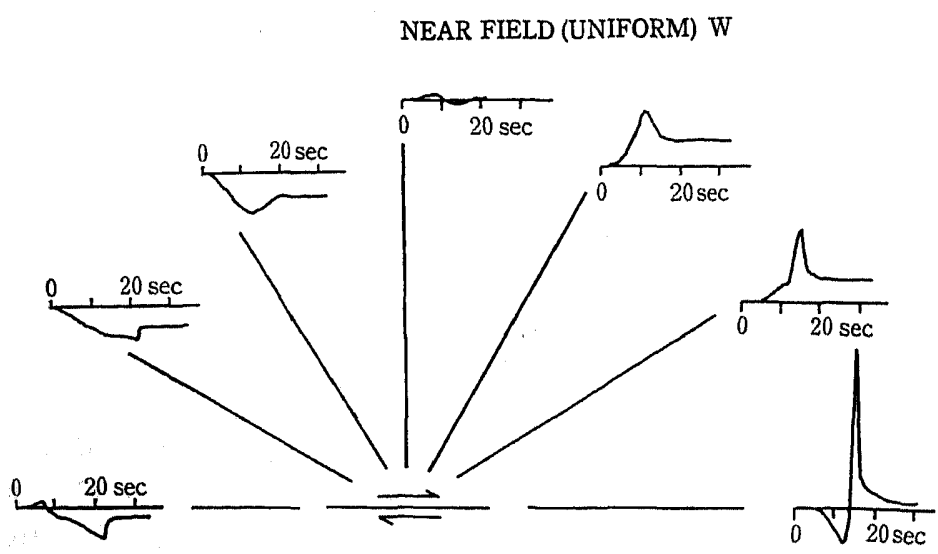

(b)

Fig. 5. Near-field seismic waves (at a distance of $50 \mathrm{~km}$ ) for uniform strength case. 
b) Line barrier (Figs. 6(a) and (b))

We can notice remarkable directively effects. At an azimuthal angle of $30^{\circ}$, the waveforms are rather flat with smaller amplitudes, in contrast to larger amplitudes and short time duration at $150^{\circ}$. The effects are particularly clear in the $z$-component waves at 0 and $180^{\circ}$.

We notice two distinct phases for the $x$-component especially at $90^{\circ}$ and for the $z$-component at 0 and $30^{\circ}$. At $180^{\circ}$, however, these sharp phases do not appear, but a phase probably due to the fracture of the barrier appears at about $19 \mathrm{sec}$. For the case of block barrier, two prominent phases also appear and their wave shapes are very similar to the case with a line barrier. Thus for our limited

NEAR FIELD (LINE) U

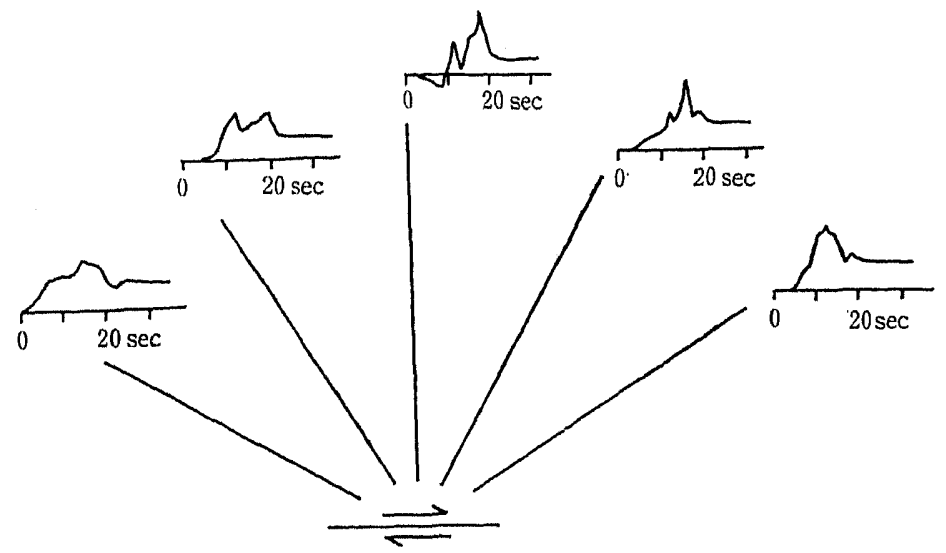

(a)

NEAR FIELD (LINE) W

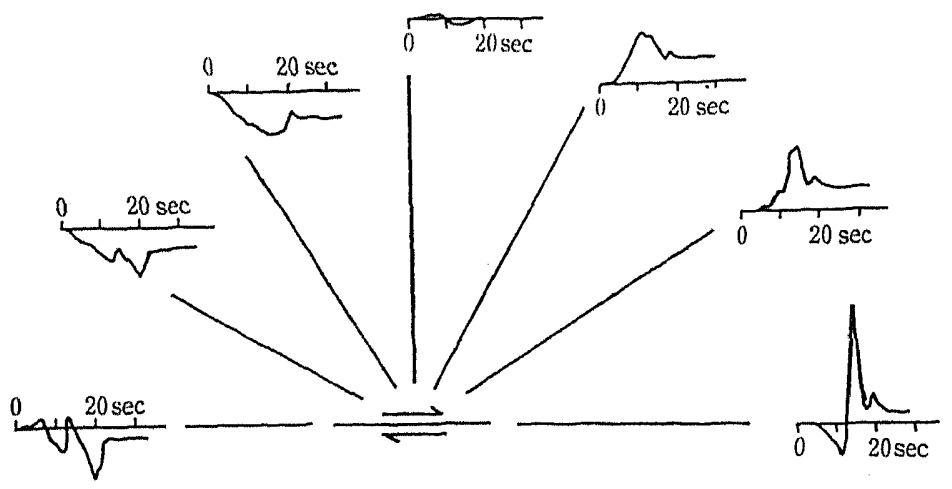

(b)

Fig. 6. Same as Fig. 5 for line barrier case (LB-1). 
examples, it is difficult to distinguish these two different types of strength distributions from seismic waveforms observed in the near-field.

c) Random strength (Figs. 7(a) and (b))

For the case of random distribution of the strength, it is noticed that high frequency waves are added, especially at 30,90 and $120^{\circ}$ for the $x$-components, and at 0 and $180^{\circ}$ for the $z$-component, respectively as might be expected.

\section{NEAR FIELD (RANDOM) U}

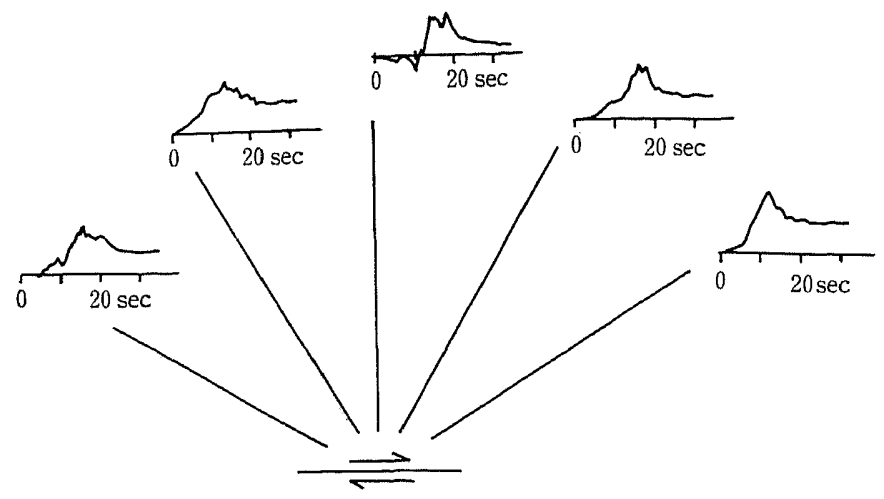

(a)

NEAR FIELD (RANDOM) W

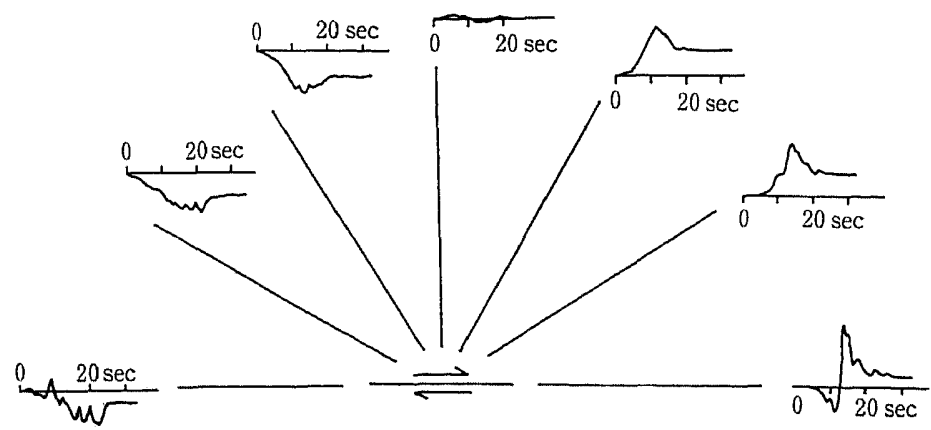

(b)

Fig. 7. Same as Fig. 5 for random barrier strength case (D-2).

\subsection{Far-field seismic waves}

We calculate the corresponding far-field seismic $\mathbf{P}$ waves radiated from frictional faults with different types of strength distribution, by the method described in Mikumo (1969). The take-off angle here is taken as $30^{\circ}$ and the azimuthal angles are taken to be $30,60,120$ and $150^{\circ}$. Here we show only vertical 
component of displacement vector referred to the coordinate taken at the fault plane, not at observation stations.

a) Uniform strength (Fig. 8)

We calculate seismic waveforms in this case mainly for later comparison with those in other cases. The calculated waves in this case do not contain short-period components. We can see some directivity effects in the waveforms. The waves emitted in the direction of rupture propagation at 120 and $150^{\circ}$ have somewhat larger amplitudes and with slightly shorter duration times in comparison with those in the opposite direction.

FAR FIELD (UNIFORM)

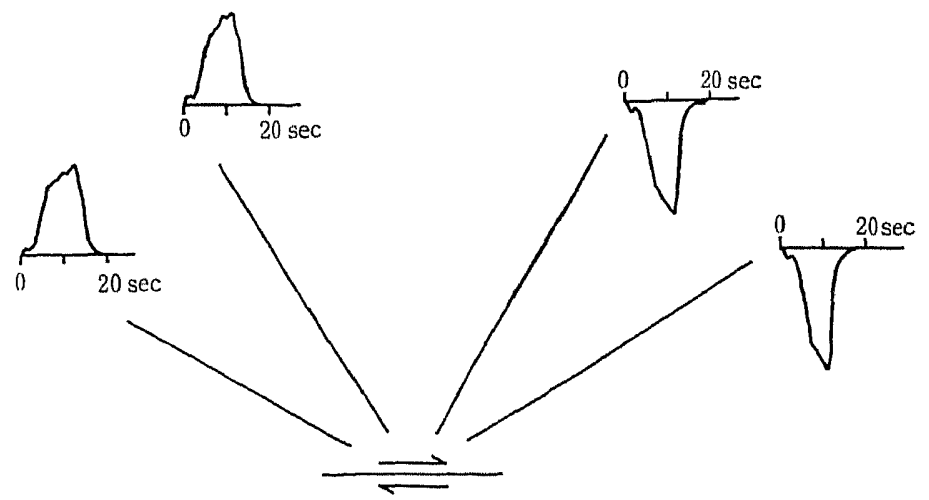

Fig. 8. Far-field seismic waves for uniform strength case.

FAR FIELD P WAVES (LINE)

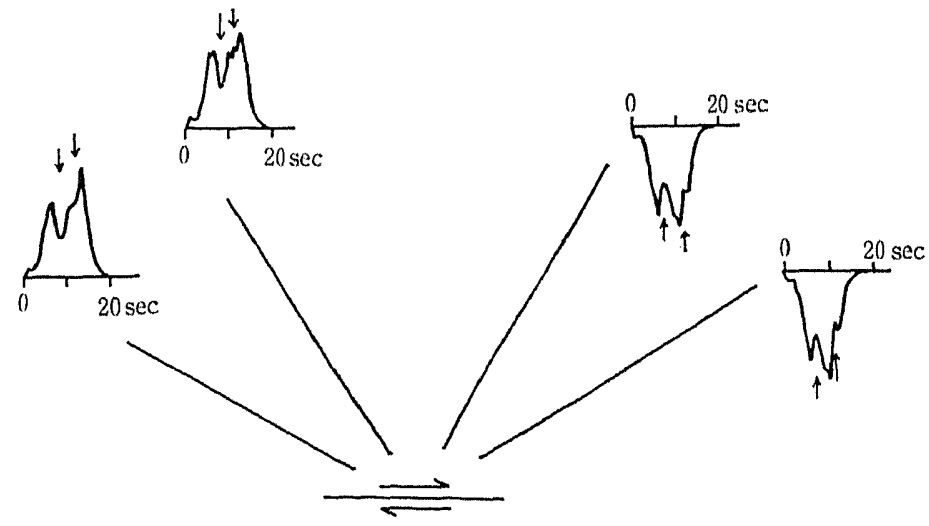

Fig. 9. Same as Fig. 8 for line barrier case (LB-1).

b) Line barrier (Fig. 9)

Far-field seismic waves from the fault with a line barrier are constituted 
by three distinct phases. We can imagine that the first arrival appears to be generated by the rupture before the rupture front reaches the barrier region, the second phase is due to the passage of the rupture on the barrier, and that the third one is caused by the fracture of the barrier. We shall examine the sources of these phases. Arrows in Fig. 9 indicate the expected arrival times of these phases, which are calculated by the following equation,

$$
\begin{aligned}
& \Delta T_{1 j}=T_{1}-\frac{l}{4} \cos \left(\frac{\pi}{6} j\right) \\
& \Delta T_{2 j}=T_{2}-\frac{l}{4} \cos \left(\frac{\pi}{6} j\right) \quad(j=1,2,4,5),
\end{aligned}
$$

where $T_{1}$ and $T_{2}$ are the times of extending rupture beyond the barrier and of fracturing the barrier region, respectively, and $l$ is half a length of the fault. We find here that sharp changes in the waveforms occur exactly on the time indicated by the arrows, and hence the sources of these phases can be explained by the above interpretations. In the case of block-barrier, we can see similar waveforms to Case b), suggesting that the rupture process is almost the same as in the former case. However, the rupture time of the barrier region occurs later than that in the case with a line barrier and then the third phase can be distinguished more clearly.

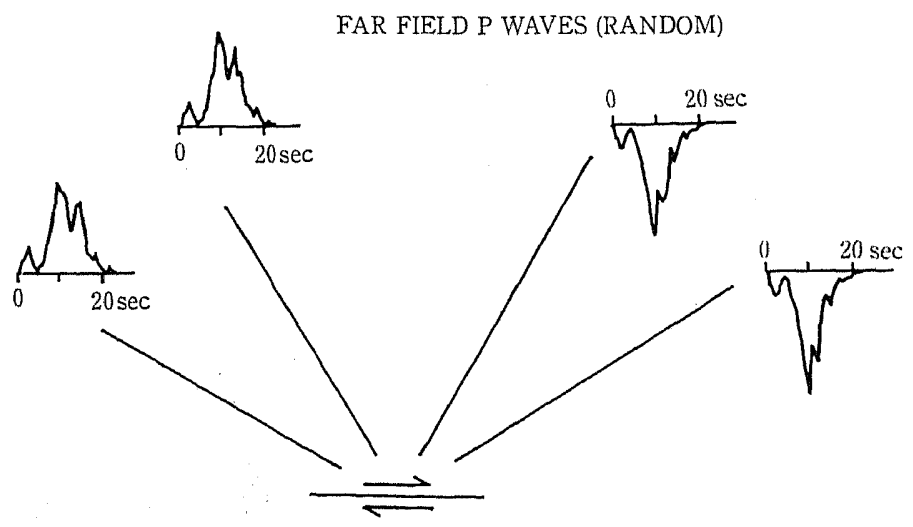

Fig. 10, Same as Fig. 8 for random barrier strength case (D-2).

c) Random strength (Fig. 10)

A fault with random strength distributions generates complex far-field seismic waves constituted by many phases. All of these phases are commonly observed at different times at different observing points. Although the rupture process in this case appears complex, this may be regarded as unilateral propagation as a whole. By comparing the wave amplitudes and periods at 30 and $150^{\circ}$, we can show these features; at $150^{\circ}$, the waves have larger amplitudes and sharp waveforms, but in comparison with smaller amplitudes with wider pulse width 
at $30^{\circ}$. These general features may well be compared with those in the case of a uniform strength.

For the case with line and block barriers, the change in the displacement on the fault plane due to the fracture of the barrier region is not so large in Part I, and it is a problem whether this change can generate any clear phase or not. In this paper, however, we have shown that this small change could radiate these phases.

\section{Spectrum}

Now, we shall discuss the spectrum of seismic waves in the near-and far-fields, which have been calculated in the former section. Spectral features of seismic waves are very important for source mechanism studies; for example, Brune's method (BRUNE, 1970) of estimating source parameters from seismic spectra has often been used by many seismologists (e.g., TUCKER and BRUNE, 1977). It has been pointed out, however, that this method is so simple that there are some difficulties in the application to actual data analysis and also in the validity of the model. These problems have been argued by some researchers (e.g., SATo and Hirasawa, 1973; BRUNe et al., 1979), particularly about possible errors in the source dimension estimated from the corner frequency. Nevertheless, only this method has been applied to source mechanism studies of small-size earthquakes, although TsukudA (1980 a, b) recently investigated the source mechanism. of micro-earthquakes by means of the time domain analysis familiar to the case of large earthquakes. We investigate here the nature of seismic spectrum particularly in relation to the change in the corner frequency for different types of strength distributions on the fault. This analysis would yield some estimate of possible variations in the corner frequency and hence the accuracy of the inferred source dimensions.

\subsection{Near-field spectrum}

In order to obtain a seismic spectrum, we apply a Hamming window to nearfield waves including both the $P$ and $S$ waves, and then Fourier-analyze the windowed signals. This implies that the calculated spectrum includes the two types of body waves which are difficult to separate in the near-field.

a) Uniform strength (Figs. 11(a) and (b))

The spectrum of the $x$ - and $z$-component displacements parallel and perpendicular to the fault plane are shown in Figs. $11(\mathrm{a})$ and (b). For the $x$-component, we obtain the corner frequency $\left(f_{\mathrm{e}}\right)$ ranging between 0.02 and $0.05 \mathrm{~Hz}$, and the decaying rate of the spectrum over high frequencies ranges from 1.2 to 1.6. Azimuthal dependences of the decay constant and of the corner frequency are not clear. The average corner frequency is approximately $0.03 \mathrm{~Hz}$ and that of the decay constant is 1.5. For the $z$-component, we also find that the corner frequency 


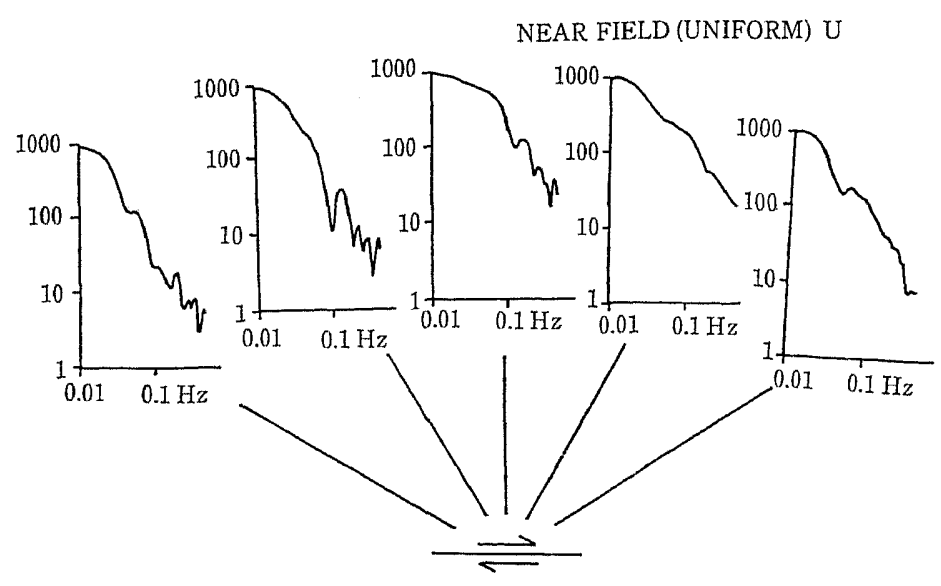

(a)

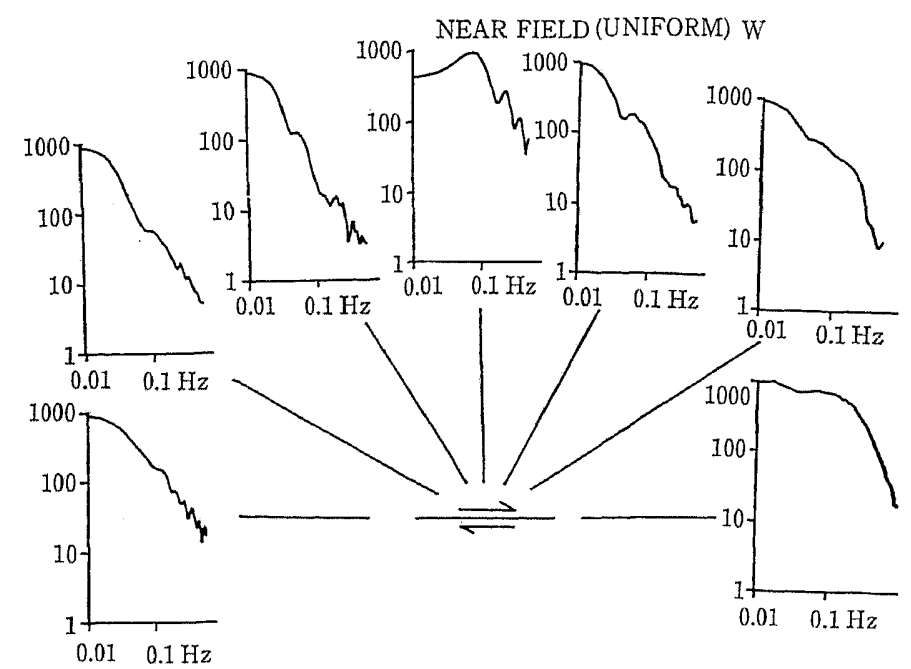

(b)

Fig. 11. Spectra of near-field seismic waves for uniform strength case.

lies in $0.02 \mathrm{~Hz}$ and the decay constant takes on a value amounting to 1.5 at 30 , 60,120 and $150^{\circ}$. For $180^{\circ}$, however, the corner frequency is extended to $0.2 \mathrm{~Hz}$ and the decay constant reaches about 2. This implies the abundance of lower frequency component relative to higher one due to progressing ruptures.

b) Line barrier (Figs. 12(a) and (b))

It is also found in this case that the corner frequency is almost $0.02 \mathrm{~Hz}$ and the high frequency decay constant falls on 1.0-1.3. The spectral shapes below $0.1 \mathrm{~Hz}$ are almost the same throughout different azimuths, and there are some troughs beyond $0.1 \mathrm{~Hz}$. 


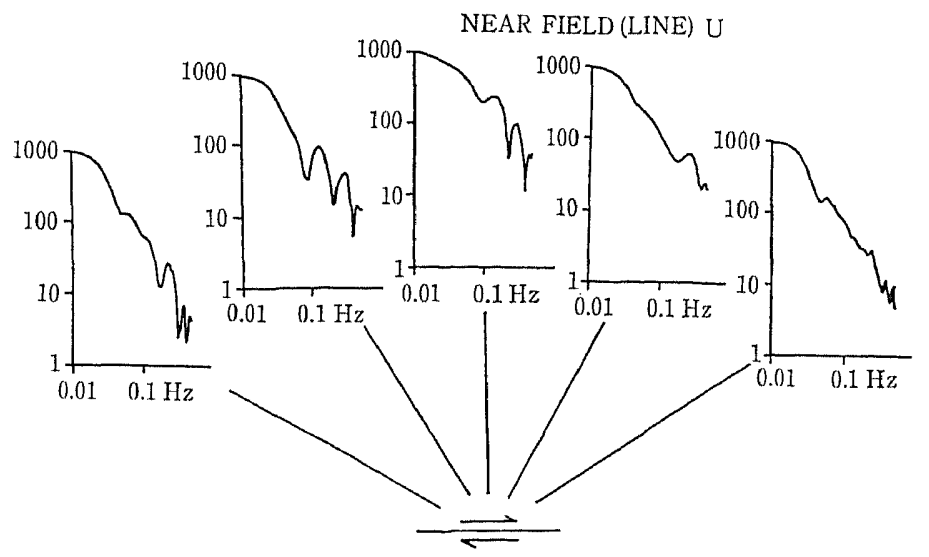

(a)

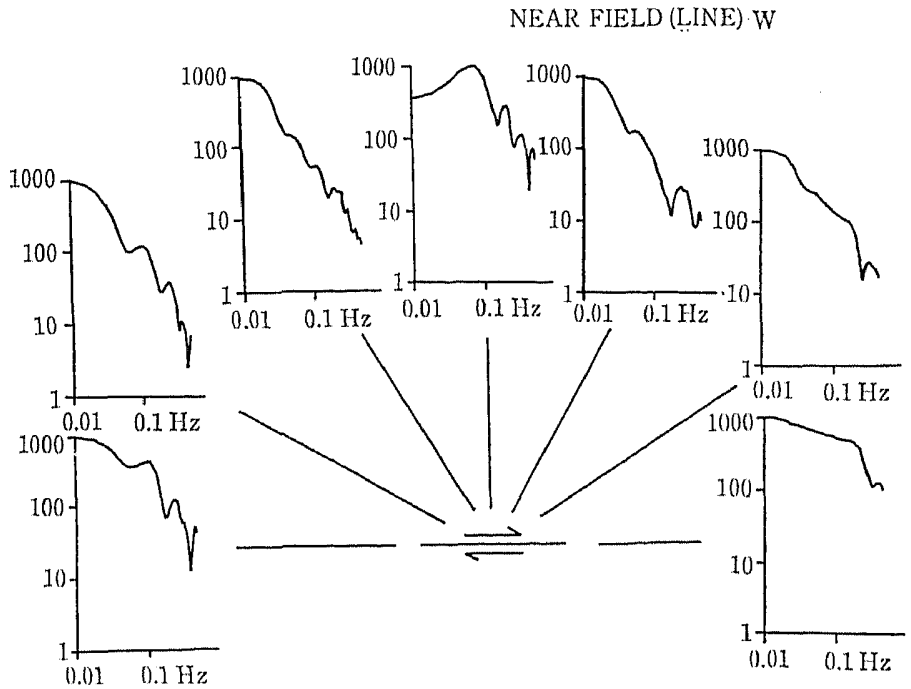

(b)

Fig. 12. Same as Fig. 11 for line barrier case (LB-1).

In the case of block barrier, we have nearly the same corner frequency and high-frequency decay constant as in the case with a line barrier, and the spectral shapes are found to be similar to those in Case b).

c) Random strength (Figs. 13(a) and (b))

The corner frequencies at different azimuths are not very different from those in Case a), but the decay constant over high frequencies is ranges from 0.8 to 1.3. These results indicate that high frequency component waves may be more effectively generated in the case of random distributions of the strength. It is to be noted that the dependence of the corner frequency on the strength distribution over the 


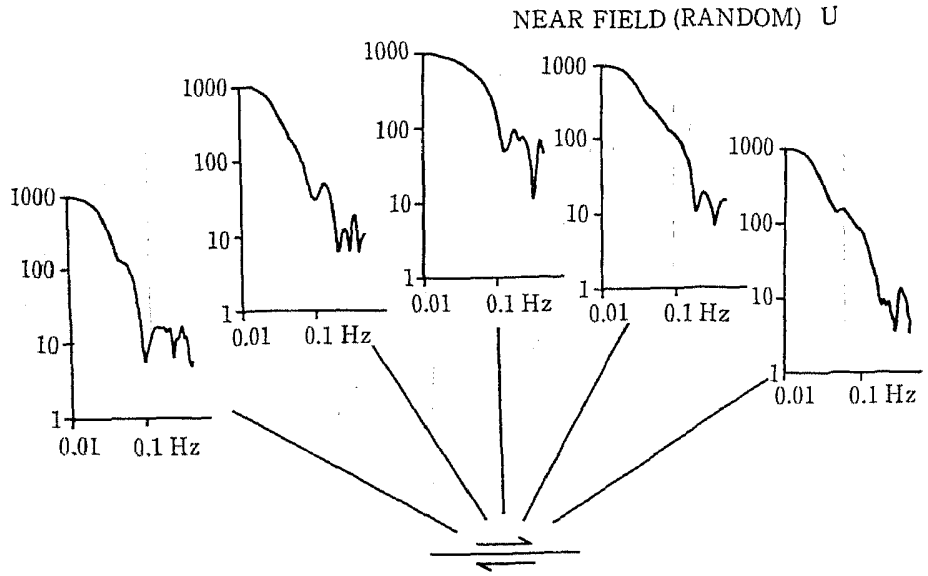

(a)

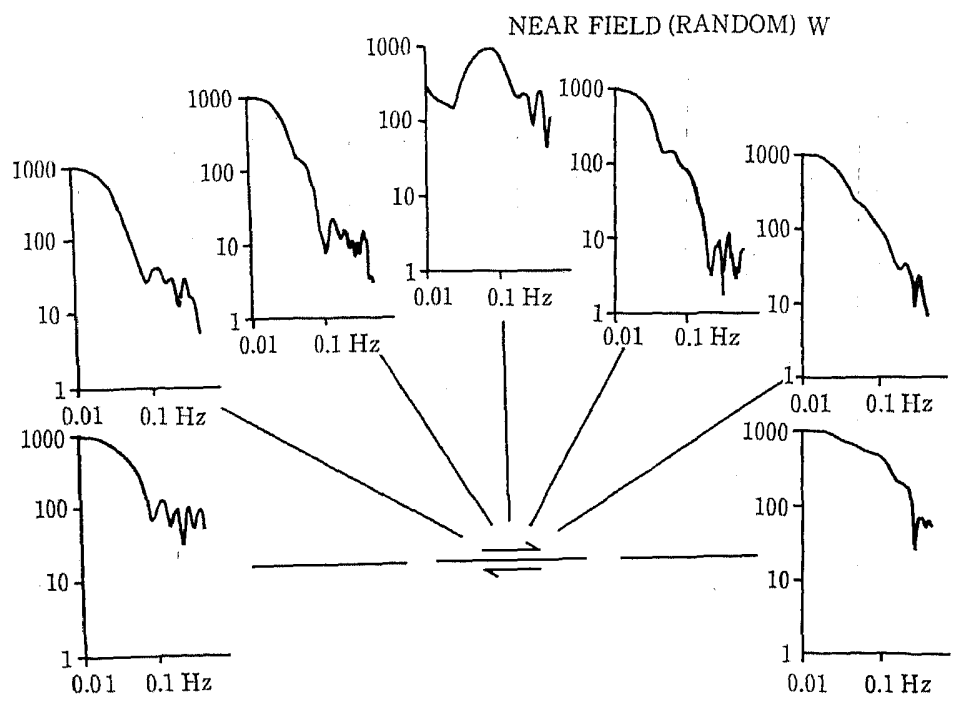

(b)

Fig. 13. Same as Fig. 11 for random barrier strength case (D-2).

fault is not so clearly defined for specific azimuths, while the decay of the spectrum over high frequencies strongly depends on the distribution. It has been demonstrated that the fault with irregularly distributed strengths radiates high frequency seismic waves in the near-field (Mrkumo and Mryatake, 1978). Das (1976) also obtained nearly the same results for far-field seismic waves in two-dimensional calculations. These results have been confirmed for near-field waves from more complete three-dimensional calculations in this paper. 


\subsection{Far-field spectrum}

The spectrum of far-field $P$ waves has been calculated in the same way for four different types of strength distributions on the fault.

a) Uniform strength (Fig. 14)

The corner frequency at all observing points in different azimuths appear to lie at nearly $0.05 \mathrm{~Hz}$ and the decaying rate of the spectrum in the high frequency range is about 2 . These results are expected from a conventional dislocation model of earthquakes (e.g., AKI, 1972; Geller, 1976).

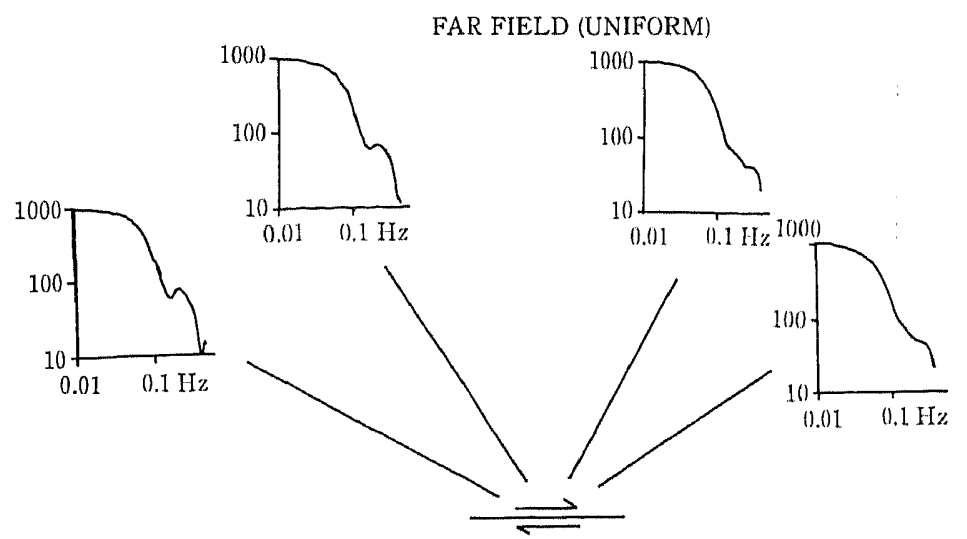

Fig. 14. Spectra of far-field seismic waves for uniform strength case.

\section{b) Line barrier (Fig. 15)}

We found in this case that the corner frequency is $0.03 \mathrm{~Hz}$ which is somewhat lower than that in Case a), and that the decay constant is estimated as about 1.2-1.4, again smaller than that for the previous case. There exists a spectral trough about $0.2 \mathrm{~Hz}$.

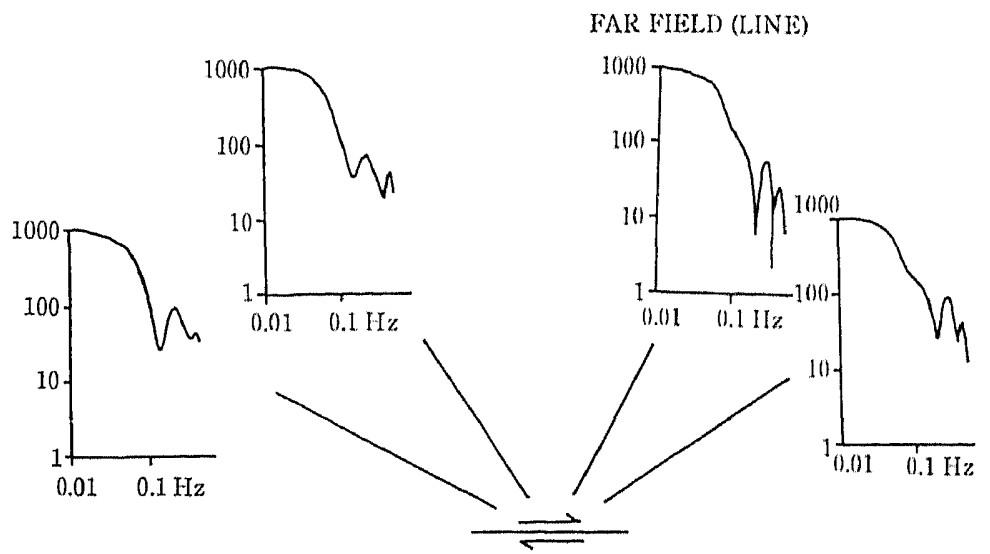

Fig. 15. Same as Fig. 14 for line barrier case (LB-1). 
In the case of block barrier, the results are almost the same as in Case b); the corner frequency is about $0.03 \mathrm{~Hz}$, and the decay constant ranges from 1.0 to 1.4. The first trough at frequencies around $0.2 \mathrm{~Hz}$ can also be noticed but in some cases deeper than that in Case b).

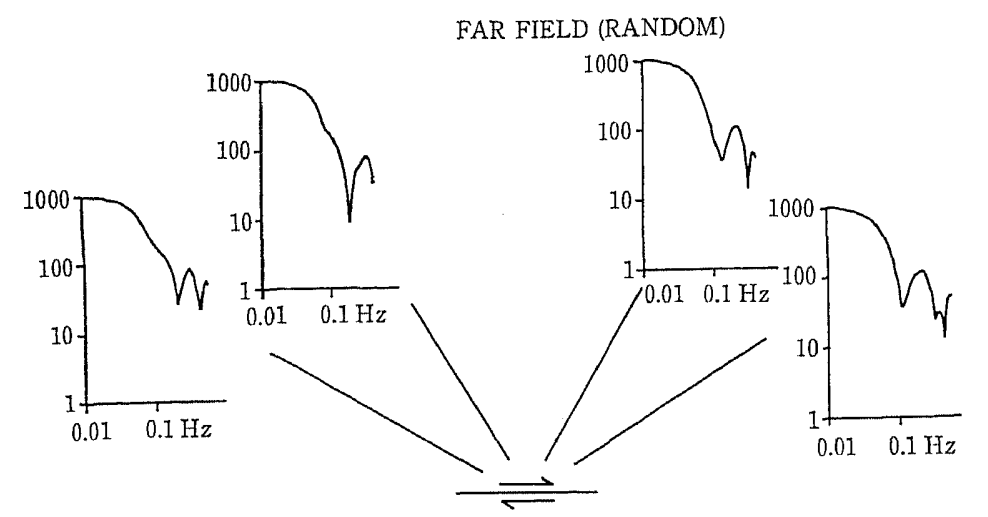

Fig. 16. Same as Fig. 14 for random barrier strength case.

\section{c) Random strength (Fig. 16)}

In this case, the corner frequency is located at about $0.03 \mathrm{~Hz}$, which is almost the same as those in Cases b) and c), whereas the decay constant decreases down to about 1.0. The latter suggests the abundance of high frequency components in the case of random distribution of strengths, which agrees with the $w$-inverse dependence in the previous results (MIKUMO and MIYATAKE, 1978). For azimuthal angles of 30 and $60^{\circ}$, a trough exists at $0.2 \mathrm{~Hz}$, while it is at about $0.1 \mathrm{~Hz}$ at other azimuthal angles. The position of the first spectral trough has been used in some cases (e.g., KHATTRI, 1969) for estimating the fault dimension. The present study for various types of strength distribution on the fault does not yield strong variations in the position of the corresponding frequency. Our present results for far-field $\mathrm{P}$ waves indicate that the corner frequency does not strongly depend on the irregularity of the fault strength and on azimuthal angles, although the dependence has been suggested for near-field $S$ waves (Mikumo and Mryatake, 1978). It should be here noticed that the spectral amplitude level at low frequencies goes down to some extent if some unruptured regions remain on the fault plane. This indicates that the seismic moment derived from the spectral amplitude will show a somewhat smaller value than that in the case of a completely ruptured fault. The reduction of the seismic moment ranges between $0.5-0.9$ in the case with high strength barriers. It might be possible to infer whether there actually exist barriers on the fault or not, on the basis of spectral amplitudes at low-frequency, the decay of high-frequency amplitude (CHOUET et al., 1978). 


\section{Conclusion}

The main results in this paper are as follows:

1) Seismic waves immediately above the fault are largely affected by the fracture of a fault segment nearest the observing point. When the rupture passes this point, we observe a sharp phase of large amplitude.

2) Complex seismic waves including multiple shocks are generated mainly by irregular propagation of the rupture, rather than by complex source time functions. This complex rupture propagation radiates high frequency seismic waves.

3) Decaying constant of near-field spectrum in the case of uniform strength, line barrier, block barrier, and random barrier are about 1.5, 1.2, 1.2, and 1.0, respectively. And corner frequencies are almost the same for all the cases.

4) Decaying constant of far-field spectrum in the case of uniform strength, line barrier, block barrier, and random barrier are about $2.0,1.5,1.5$, and 1.0, respectively.

I would like to express my deepest gratitude to my advisor Professor Takeshi Mikumo for his continuous encouragements, critically reading the manuscript and giving many valuable suggestions.

I would like to thank Professor Haruo Miki and Dr. Hikaru Watanabe for their useful suggestions and discussions. Thanks are also due to Dr. Torao Tanaka for his suggestion and helpful discussions. Discussions with Dr. Yoshinori Iwasaki were helpful. Discussions with the members of Disaster Prevention Research Institute, Kyoto University and Earthquake Research Institute, University of Tokyo were helpful.

The numerical computations were carried out by FACOM M-200 at the Data Processing Center, by FACOM M-160 at computer room of Chemical Research Institute, and by FACOM M-140 at computer room of Disaster Prevention Research Institute, Kyoto University.

Finally I thank to my colleagues, Mrs. Sumiko Hiyama, Miss Yoshiko Kotake and Mr. Ikki Kohketsu of Earthquake Prediction Data Center, Earthquake Research Institute, University of Tokyo for their encouragements.

This is a part of Ph.D. thesis submitted to Kyoto University.

\section{REFERENCES}

AKI, K., Scaling law of seismic spectrum, J. Geophys. Res., 72, 1217-1231, 1967.

AkI, K., Scaling law of earthquake time-function, Geophys. J. R. Astron. Soc., 31, 3-25, 1972. Ben-Menahem, A., Earthquake similarity laws, Phys. Earth Planet. Inter., 15, 10-18, 1977.

BRUNE, J. N., Tectonic stress and the spectra of seismic shear waves from earthquakes, J. Geophys. Res., 75, 4997-5009, 1970.

Brune, J. N., R. J. ARchuleta, and S. Hartzell, Far field S wave spectra, corner frequency, and pulse shapes, J. Geophys. Res., 84, 2262-2272, 1979.

Chouet, B., K. Akr, and M. Tsujuru, Regional variation of the scaling law of earthquake source spectra, Bull. Seismol. Soc. Am., 68, 49-79, 1978.

DAs, S., A numerical study of rupture propagation and earthquake source mechanism, Ph.D. thesis of M.I.T., 1976.

DAS, S. and K. AKI, A numerical study of two dimensional spontaneous rupture propagation, Geophys. J. R. Astron. Soc., 50, 643-668, 1977a. 
DAS, S. and K. AKI, Fault planes with barriers: A versatile earthquake model, J. Geophys. Res., 82, 5648-5670, 1977b.

GELLER, R. J., Scaling relations for earthquake source parameters and magnitudes, Bull. Seismol. Soc. Am., 66, 1501-1522, 1976.

HASKELL, N., Total energy and energy spectral density of elastic wave radiation from propagating faults. 2. A statistical source model, Bull. Seismol. Soc. Am., 56, 125-140, 1966.

KANAMORI, H. and D. L. ANDERSON, Theoretical basis of some empirical relations in seismology, Bull. Seismol. Soc. Am., 65, 1075-1096, 1975.

KHATTRI, K., Determination of earthquake fault plane, fault area, and rupture velocity from the spectra of long period P waves and the amplitude of SH waves, Bull. Seismol. Soc. Am., $59,615-630,1969$.

Maruyama, T., On the force equivalents of dynamic elastic dislocations with reference to the earthquake mechanism, Bull. Earthq. Res. Inst., Univ. Tokyo, 41, 467-486, 1963.

Mikumo, T., Long-period $\mathbf{P}$ waveforms and the source mechanism of intermediate earthquakes, J. Phys. Earth, 17, 169-192, 1969.

MiKumo, T. and T. MiYATAKE, Dynamical rupture process on a three-dimensional fault with nonuniform frictions and near-field seismic waves, Geophys. J. R. Astron. Soc., 54, 417-438, 1978.

MIYATAKE, T., Numerical simulations of earthquake source process by three-dimensional crack model. Part I. Rupture process, J. Phys. Earth., 28, 565-598, 1980.

SATO, T. and T. HiRASAWA, Body wave spectra from propagating shear cracks, J. Phys. Earth, 21, 415-431, 1973.

TsUKUDA, T., Source process of microearthquakes deduced from $\mathrm{P}$ waveforms. Part I. Waveform analysis and source modeling, submitted to J. Phys. Earth, 1980a.

TsuKuDA, T., Source process of microearthquakes deduced from P waveforms. Part II. Source parameters and the structure of the fractured region within the crust, submitted to J. Phys. Earth., $1980 \mathrm{~b}$.

TUCKER, B. E. and J. N. BRUNE, Source mechanism and mb-Ms analysis of aftershocks of the San Fernando earthquake, Geophys. J. R. Astron. Soc., 49, 371-426, 1977. 